\title{
On further Subdivisions of Regular Polygons using Structures other than Spidrons and Tiling Patterns Generated by them
}

\author{
T. Gangopadhyay \\ XLRI \\ C.H.Area (E), Jamshedpur, \\ India
}

\begin{abstract}
A regular $n$-sided polygon can be split into $n \mathrm{n}$-part spidrons, as well as, into n-part ladders. In the present paper, it is shown that there exist yet other linked triangular structures which are distinct from spidrons and ladders and which can also be used to subdivide regular polygons. Tiling patterns using such subdivisions are also explored in detail.
\end{abstract}

\section{General Terms}

Tiling, Algorithm, Turbo C++, Program.

\section{Keywords}

Spidron, ladder, creeper, polygon, isosceles, subdivision

\section{INTRODUCTION}

An n-part spidron is a figure in plane geometry consisting entirely of an alternating sequence of two isosceles triangles, each with its own base angles[2], [6], [8]. The two triangles are juxtaposed in such a way that together they from a third bigger triangle. The sequence is then drawn at an appropriate angle on a smaller scale and the process is repeated ad infinitum. n-part spidrons have been largely studied for $n=6$ and 8[5]. A regular $\mathrm{n}$-sided polygon can be split into $\mathrm{n} n$-part spidrons[7]. An 8-part spidron is displayed in Figure 1. Erdely[2], has called the same structure a semispidron.

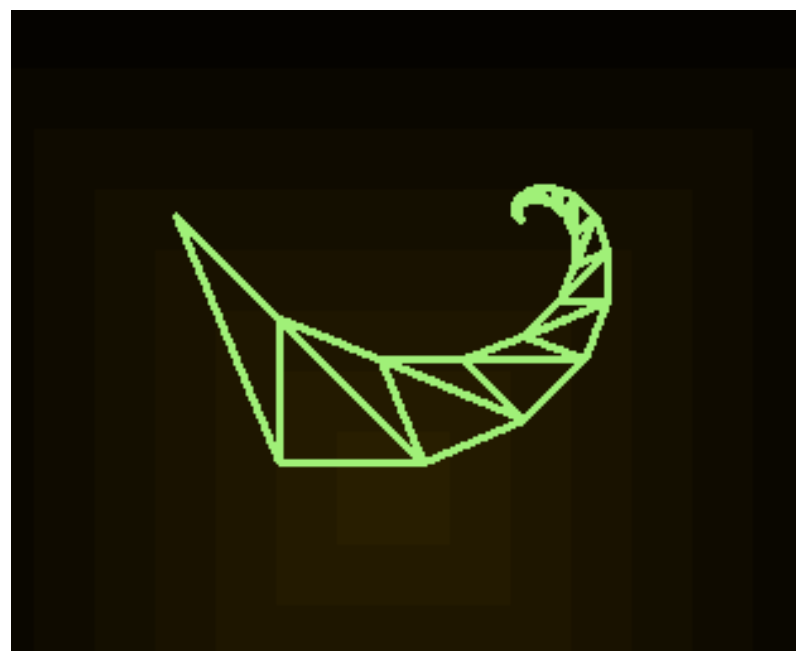

Fig 1 : An eight-part spidron

In earlier papers, the present author has developed an alternate way of constructing individual n-part spidrons so as to algorithmically juxtaposing them to produce a regular $\mathrm{n}$-sided polygon (Gangopadhyay([3]) and has also shown that there exist other linked triangular structures such as ladders(Figure 2) which are distinct from spidrons and which can also be used to subdivide regular polygons(Gangopadhyay([4]). In the present paper, it is shown that there exist yet other linked triangular structures which are distinct from spidrons and ladders and which can also be used to subdivide regular polygons. Tiling patterns using such subdivisions are also explored in detail. These are primarily the distinctive features of this paper.

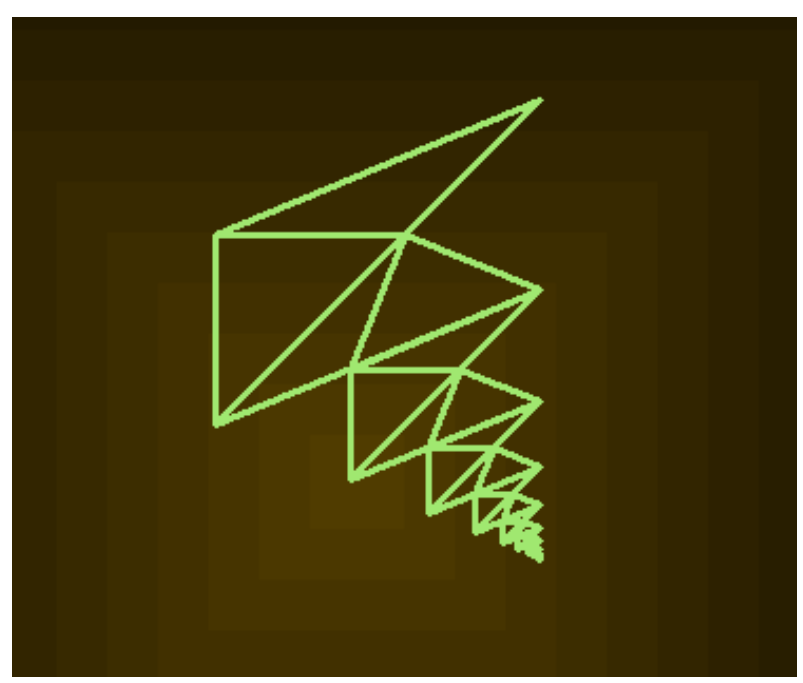

Fig 2 : An eight-part ladder

\section{THE ALGORITHM}

The basic building blocks for subdividing a regular polygon are linked triangular structures that are drawn recursively on a reduced scale. The process is similar to that of constructing a spidron or a ladder, though the final outcome is quite distinct.

The algorithm is best explained in terms of Figure 3, which depicts two juxtaposed isosceles triangles, this being the basic building block of a spidron. Let the base angle $A B C$ have value ag and let $A B$ have length $s$. Then $A C=C D=A B=s$ and angle $\mathrm{ACB}=\mathrm{ag}$. Also angle $\mathrm{CAD}=$ angle $\mathrm{ADC}$.From this it follows that angle $\mathrm{CAD}=$ angle $\mathrm{ACD}=\mathrm{ag} / 2$. Also $\mathrm{Bc}=2 \mathrm{~s}(\cos (\mathrm{ag}))$ and $\mathrm{AD}=2 \mathrm{~s}(\cos (\mathrm{ag} / 2))$. With this it is easy to construct the two triangles in Figure 2. The construction described above is identical to the one described in the first step of section 2 in Gangopadhyay[3]. It is to be noted that for the exact subdivision of an $n$-sided regular polygon, $a g=360 / n$. For the purposes of illustration, $n=8$ in Figures 2 to 4 . 


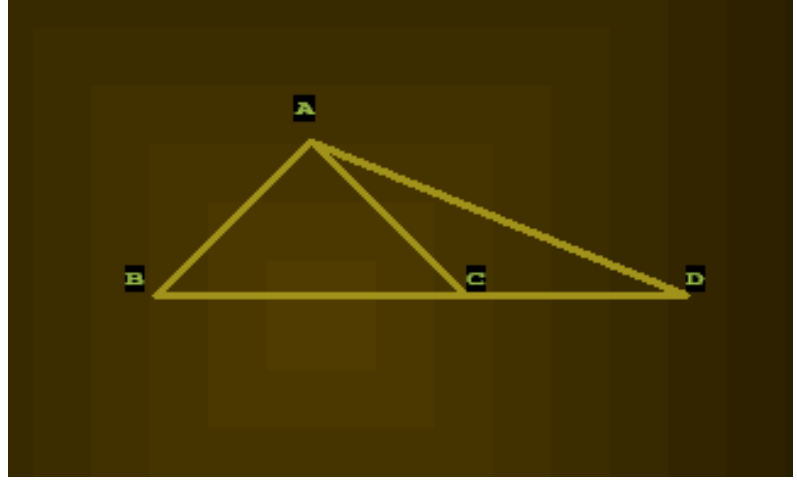

Fig 3 : The first iteration

For the second iteration one notes that point $\mathrm{A}$ is replaced by point $\mathrm{C}$ and $\mathrm{s}$ is replaced by $\mathrm{s}(\cos (\mathrm{ag}) / \cos (\mathrm{ag} / 2))$. Also, angle $\mathrm{BCE}=\mathrm{ag} / 2$ Figure 4 shows the result after the second iteration.

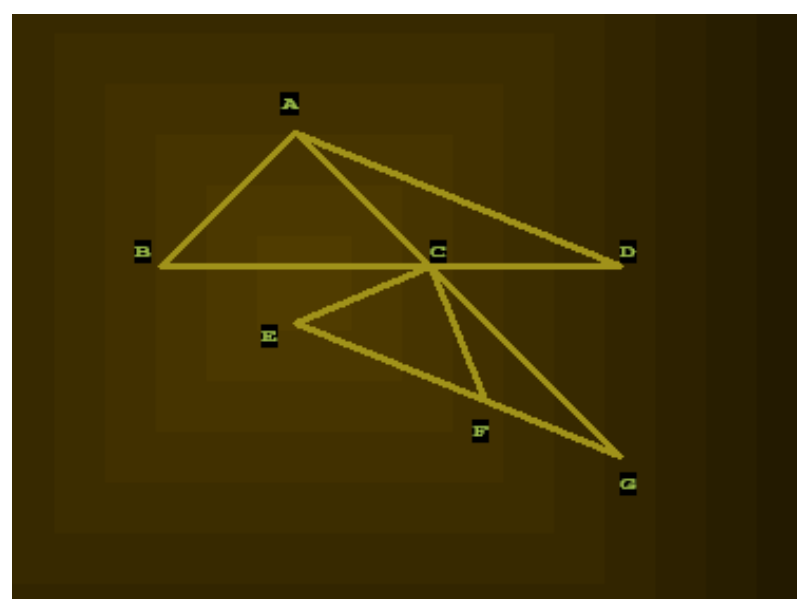

Fig 4 : The second iteration

The final result after 12 iterations is shown in Figure 5. It is to be noted that is quite distinct from the 8-part spidron shown in Figure 1as well as the 8-part ladder shown in Figure 2. For convenience, the linked triangular structure in Figure 5 is named a 'creeper'.

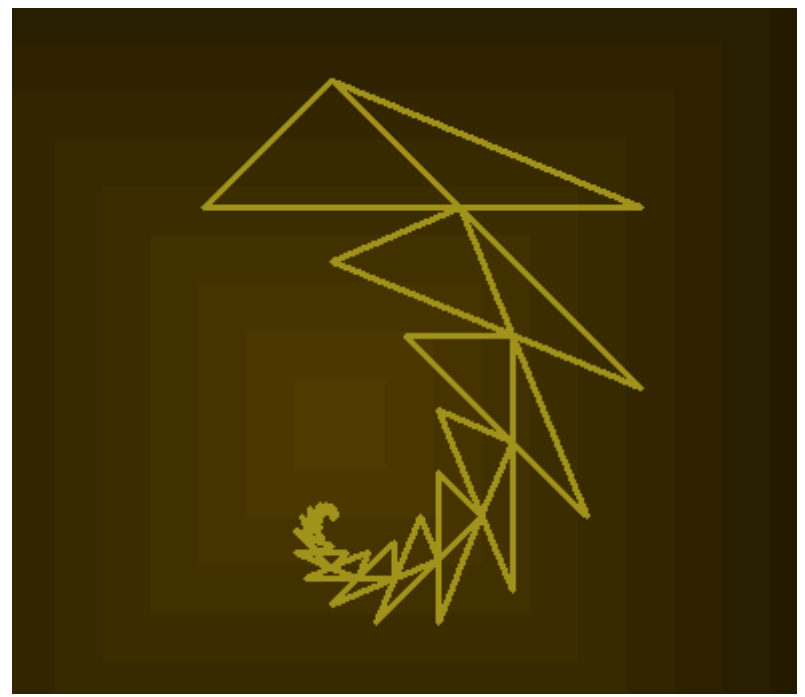

Fig 5 : The Final result
In the next section one submits a programming code in Visual $\mathrm{C}++$ that captures the algorithm and generates figures 2,3 and 4

\section{THE CODE}

The code uses a function spid which is declared first. The function has two parameters $-\mathrm{s}$, which gives the value of the two equal sides of the first isosceles triangle and 1 , which specifies the number of recursive iterations. For $s=90$ and $l=0$, 1 and 15, the output of spid is given respectively in Figures 2, 3 and 4 . spid 2 to create the superimposed polygonal design displayed in figure 4 . The functions $\mathrm{fd}$, rt, lt, pu and $\mathrm{pd}$ are adapted from turtle graphics[1] and have their usual connotations. The function fd draws a line of specified length, rt and lt respectively rotates the pen right and left by a specified angle, pu puts the pen up(no drawing) and pd puts the pen down. Finally, the for loop in the 'main' segment of the code draws $n$ ladders of the type shown in Figure 4 in a nested manner so that together they make up an n-sided regular polygon. The various functions and the code are given below:

float ang $=90, p x, p y, p s=1$;

void fd(float dist)

\{float $\mathrm{hx}=\cos ($ ang $* 3.1415926536 / 180)$;

float hy $=\sin ($ ang $* 3.1415926536 / 180)$;

float $\mathrm{nx}=\mathrm{px}-\mathrm{hx} *$ dist;

float ny=py-hy*dist;

if(ps!=1)goto label;

line(px,py,nx,ny);

label:px=nx;py=ny;

\}

float rt(float 1)

$\{$ ang+=1;

return ang; \}

float lt(float 1)

$\{$ ang- $=1$;

return ang; $\}$

void $\mathrm{pu}()$

$\{\mathrm{ps}=0 ;\}$

void $\operatorname{pd}()$

$\{\mathrm{ps}=1 ;\}$

int $\mathrm{n}=8$;float ag=360/n;

void spid(float,int);

void spid(float s,int 1$)$

$\{$ if $(1==0)\{\mathrm{fd}(\mathrm{s}) ; 1 \mathrm{t}(180-\mathrm{ag}) ; \mathrm{fd}(\mathrm{s}) ; \mathrm{fd}(2 * \mathrm{~s} * \cos (\mathrm{ag} * 3.14 / 180))$;

lt(180-ag/2); fd( $2 * \mathrm{~s} * \cos (\mathrm{ag} / 2 * 3.14 / 180))$;

lt(180-ag/2;fd(s); rt(180-ag); return;\}

$\operatorname{spid}(\mathrm{s}, 0) ; \operatorname{lt}(\mathrm{ag} / 2)$;

$\operatorname{spid}(\mathrm{s} * \cos (\mathrm{ag} * 3.14 / 180) / \cos (\mathrm{ag} / 2 * 3.14 / 180), 1-1)$;

\}

void main()

initwindow(1000,800);

float $s=90$;

$\mathrm{px}=650, \mathrm{py}=550$;

setlinestyle(SOLID_LINE,0,THICK_WIDTH); 
for $($ int $\mathrm{i}=0 ; \mathrm{i}<\mathrm{n} ; \mathrm{i}++)$

\{ setcolor $(\mathrm{i} * 5)$;

float $\mathrm{a}=\mathrm{px}, \mathrm{b}=\mathrm{py}$;

$\operatorname{spid}(\mathrm{s}, 1) ; \mathrm{px}=\mathrm{a}, \mathrm{py}=\mathrm{b} ; \mathrm{lt}(1 * \mathrm{ag} / 2+\mathrm{ag} / 2)$;

$\mathrm{pu}() ; \mathrm{fd}(2 * \mathrm{~s} * \cos (\mathrm{ag} / 2 * 3.14 / 180)) ; \mathrm{pd}() ; \mathrm{lt}(\mathrm{ag}+\mathrm{ag} / 2)$;

$\operatorname{pd}() ;\}$

$\operatorname{getch}()$;

closegraph();

\}

The output of the sample code is illustrated in Figure 6 where $\mathrm{n}$ has been set to 8 , showing how a regular octagon can be subdivided into 8 creepers.

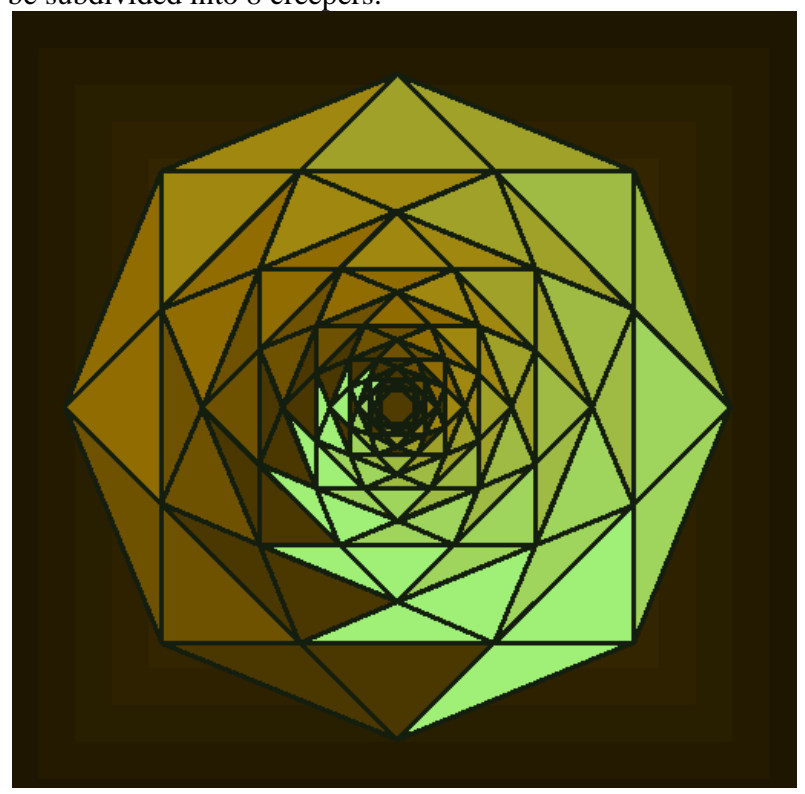

Fig 6: Output of the sample code, $n=8$

Figures 7 and 8 depict the respective outputs for $n=6$ and $\mathrm{n}=12$.

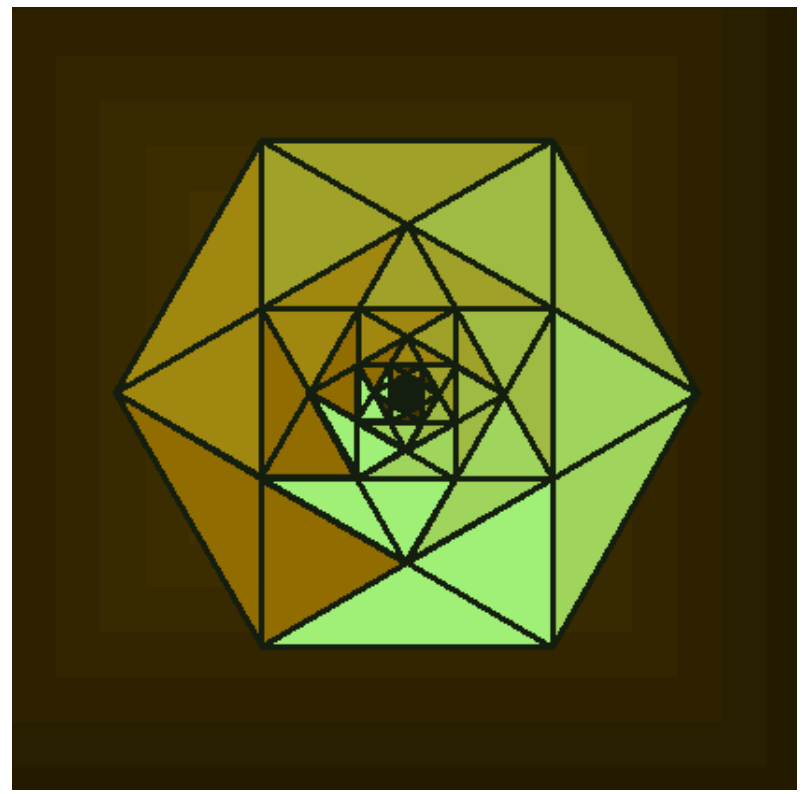

Fig. 7 : Output of the sample code for $n=6$

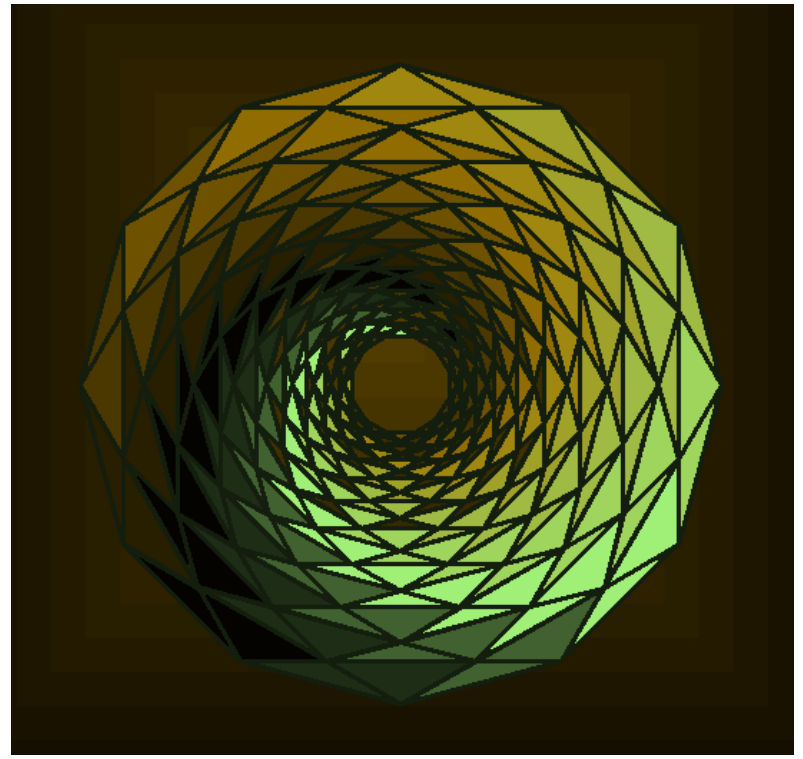

Fig. 8 : Output of the sample code for $n=12$

\section{TILING THE PLANE USING THE SUBDIVIDED POLYGONS}

Tiling with regular polygons that have been subdivided through creepers offer several interesting new patterns. These are depicted in the Figures 8-12. Figures 8-10 show a tiling patterns that uses the subdivided regular hexagon in Figure 6 as the basic building block. The designs differ owing to the alternate methods of coloring individual creepers. Figures 11 and 12 depict two tiling patterns both created by using the subdivided regular octagon in Figure 5 as the basic building block, In Figure 11 the octagons just touch each other, while in Figure 12 they intersect.

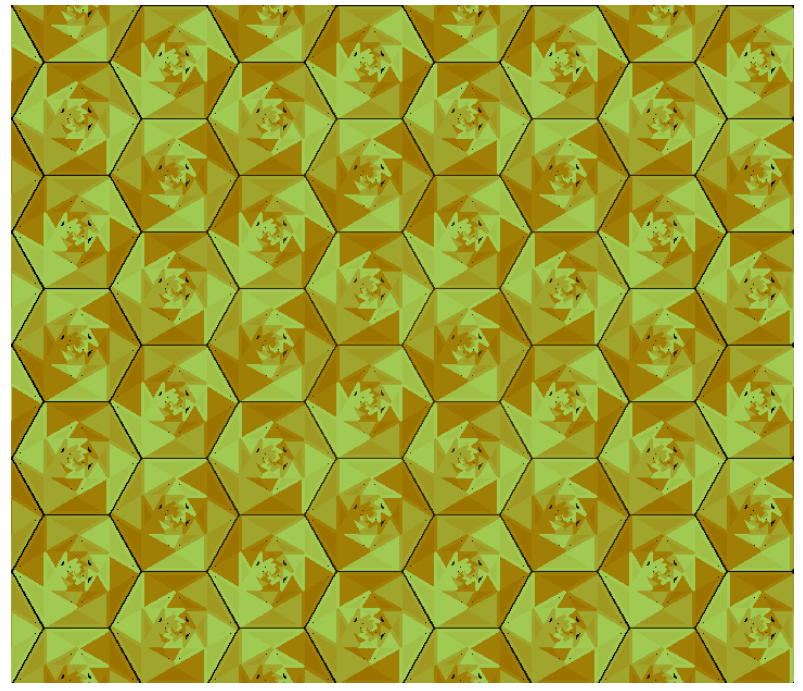

Fig. 8 : Tiling pattern-1 with subdivided regular hexagons 


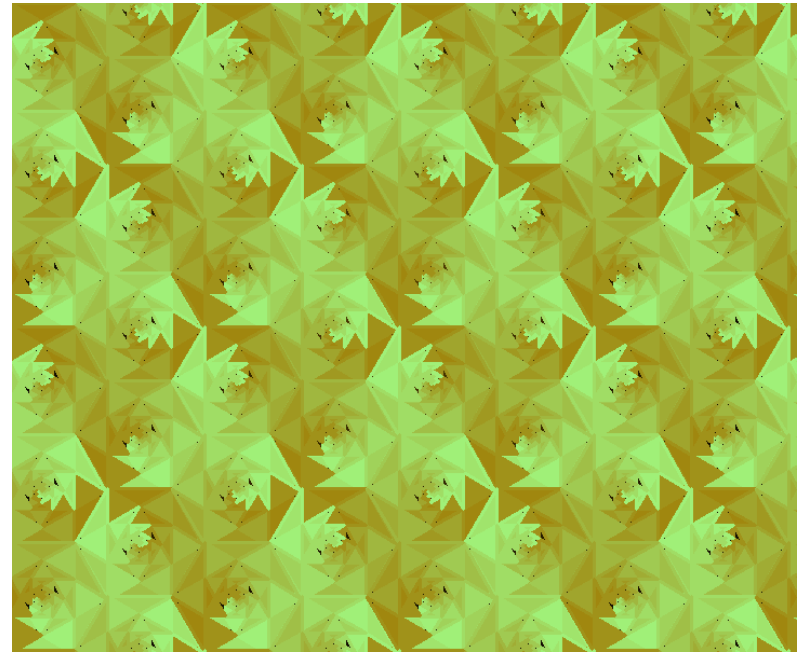

Fig. 9 : Tiling pattern-2 with subdivided regular hexagons

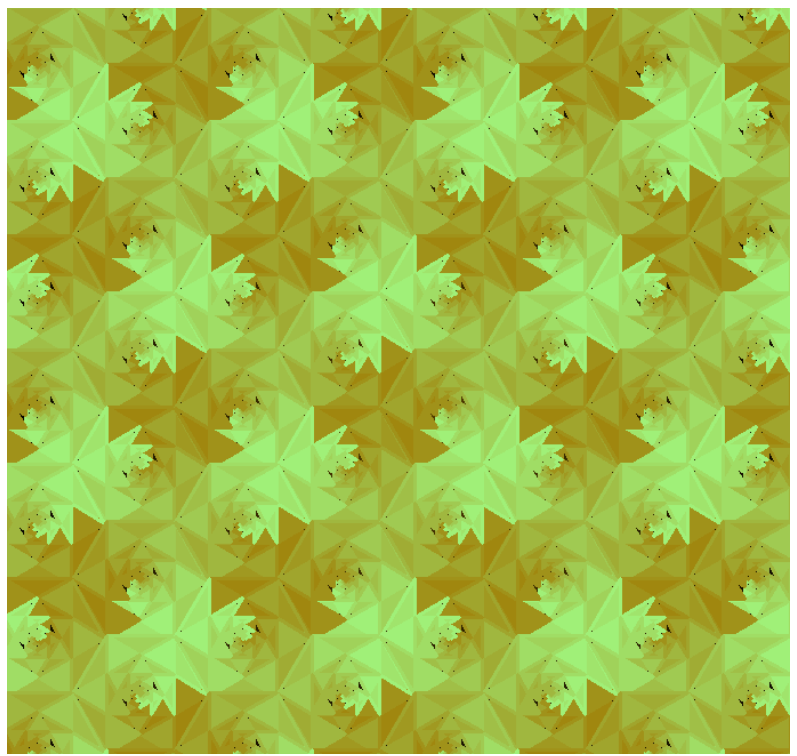

Fig. 10 : Tiling pattern-3 with subdivided regular hexagons

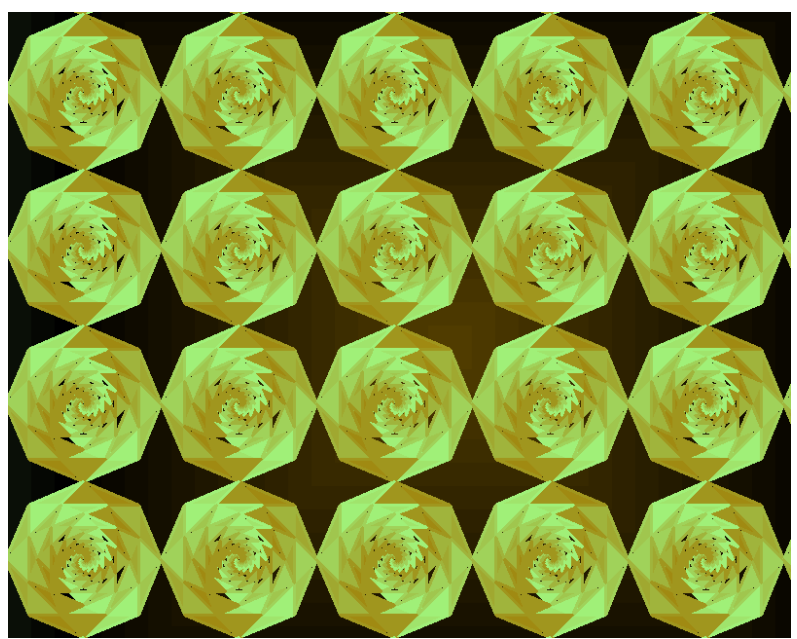

Fig. 11 : Tiling pattern with subdivided regular octagons

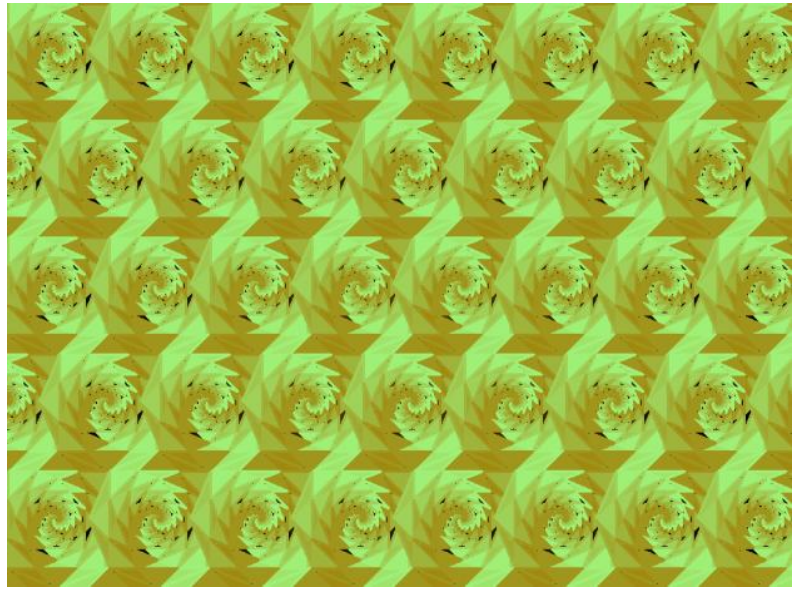

Fig. 12 : second tiling pattern using subdivided regular octagons

\section{CONCLUSION}

This paper presents the construction of a new linked triangular structure called creeper and shows how regular polygons can also be subdivided using the same. Also tiling patterns using subdivided regular polygon are explored here. In subsequent studies one will explore other such linked triangular structures which the effect of these structures on tiling patterns will also be studied. These are the aspects that would be explored in future work.

\section{ACKNOWLEDGMENTS}

The author wishes to acknowledge his debt to the referee(s) for their constructive suggestions and encouragement

\section{REFERENCES}

[1] Abelson and diSessa, Turtle Geometry, MIT Press, 1992

[2] Erdely, D. http://www.bridgesmathart.org/art exhibits/bridges2007/erdely.html.

[3] Gangopadhyay, T. On an alternate construction method for generating spidrons and new tiling patterns generated by them, International journal of Computer Applications, Volume 160, number 3, 2017.

[4] Gangopadhyay, T. On subdividing regular polygons using structures other than spidrons and tiling patterns generated by them, submitted for publication.

[5] Jacques, F. http://polyspidrons.over-blog.com/article4823990.html.

[6] Peterson, I. "Swirling Seas, Crystal Balls". ScienceNews.org. Archived from the original on February 28, 2007. Retrieved 2007-02-14.

[7] Stenzhorn, S. Mathematical description of Spidrons, http://stefanstenzhorn.com/Spidrons.

[8] Spidron From Wikipedia, the free encyclopedia https://en.wikipedia.org/wiki/Spidron. 